\title{
Advances in the Management of Pancreatic Adenocarcinoma
}

\author{
Presented by Eileen M. O’Reilly, MD
}

\begin{abstract}
Outcomes in pancreatic cancer are improving. The beneficial effects being achieved with adjuvant and neoadjuvant therapies, and the recent application of molecular profiling, both germline and somatic, are collectively impacting survival. The NCCN Guidelines for Pancreatic Cancer urge clinicians to undertake "agnostic" germline testing for all persons with pancreatic cancer. Fit patients should also be considered for adjuvant therapy with modified FOLFIRINOX (leucovorin, 5-FU, irinotecan, oxaliplatin). Novel therapies that focus on DNA damage repair strategies are proving to be important, but notably several late-stage trials of several other approaches, reported in the last year, proved disappointing.

Pancreatic cancer ranks disproportionally high as a leading cause of cancer-related death, relative to its incidence. Nonetheless, important positive signs are emerging, according to Eileen M. O'Reilly, MD, the Winthrop Rockefeller Endowed Chair in Medical Oncology, Memorial Sloan Kettering Cancer Center. Dr. O'Reilly is also head of Hepatopancreaticobilary and Neuroendocrine Cancers and Medical Codirector of the David M. Rubenstein Center for Pancreatic Cancer Research.

"In 2020, for the first time, among all-comersdiagnosed at all stages-10\% will reach the 5-year survival mark. Clearly, this is not enough, but it's a notable improvement," Dr. O'Reilly noted in her overview of pancreatic cancer at the NCCN 2020 Virtual Annual Conference.

\section{Therapeutic Landscape in $\mathbf{2 0 2 0}$}

Cytotoxic chemotherapy, especially combinations, remains the mainstay of treatment, although targeted agents and immunotherapy are entering the armamentarium. The value of DNA-damaging agents in the subset of patients with alterations in DNA damage repair genes is also now clear, driving the need for molecular profiling.

"As one refines a treatment choice for a given patient, ideally one is thinking about having somatic profiling and germline testing performed, for its treatment implications,” Dr. O’Reilly said.

\section{Somatic and Germline Testing}

Perhaps the most exciting step forward in pancreatic cancer comes from the application of molecular testing. Pancreatic cancer is dominated by a series of mutations in key driver oncogenes, most notably KRAS, p53, CDKI $N 2 A$, and SMAD4. The remainder of the genome is characterized by less frequent alterations; many involve DNA damage repair genes, for which there are treatment implications.

"It is critically important to think about getting molecular profiles, because many drivers in the tumor are theoretically targetable on the basis of drugs in trials, or by consideration of off-label use of drugs shown to be of value in other settings," she said. Examples are rare fusions in ROS, ALK, NTRK, FGFR2, and NRG1, which are identified by somatic testing. Although KRAS is seen in $90 \%$ of tumors, unfortunately, a targeted agent is not available for this mutation at this time, except for the rare subset of patients with KRAS 12C.

The rate of germline pathogenic mutations is also significant in this disease, and approximately $40 \%$ of patients harboring them would not have met guidelines for testing in the past based on traditional criteria of family or personal history of malignancy (lacking susceptibility factors). "This has led to the consideration of universal germline testing in pancreatic cancer," she said.

In a large cohort of patients from Memorial Sloan Kettering (MSK) (enriched for Eastern European Ashkenazi heritage) the dominant germline mutation was found to be $B R C A 2$, followed by $B R C A 1 .{ }^{1}$ Half of the lowerfrequency alterations were in DNA damage repair genes, "hinting at the potential for treatment opportunities," Dr. O’Reilly continued.

Germline (and somatic) testing also reveals microsatellite instability or mismatch repair deficiency (MMR-d) 
in approximately $1 \%$ of patients. ${ }^{2}$ Although this subgroup is rare, she emphasized, "It's critically important to identify them because of the implications of treatment [with checkpoint inhibitors], and to identify other family members possibly carrying an underlying germline mutation."

With these points in mind, the NCCN Guidelines for Pancreatic Adenocarcinoma now recommend agnostic germline testing for all patients with pancreatic cancer, independent of stage, age, heritage, and personal or family history. The guidelines also recommend the use of a multigene panel rather than a traditional hierarchical gene testing approach. Somatic profiling (preferably tumor-based, not blood-based) can be considered for patients with advanced disease who are candidates for further treatment.

\section{Models of Genetic Testing}

Genetic testing can be performed in several ways. At MSK, consenting patients are shown a pretest educational video along with an educational discussion. Test results are communicated by the treating physician, and patients with germline alterations are referred to a genetics counselor for follow-on completion of genetic testing.

"This approach has revolutionized our ability to do efficient testing, provide access to more patients and to have relatively quick results," Dr. O’Reilly shared.

Testing can be done with a commercial panel, an inhouse panel, or the BRACAnalysis CDx approved with olaparib; direct-to-consumer testing is not advised. The test should at least encompass the minimum 10 to 12 core genes known to be associated with predisposition or to be targetable.

\section{Therapeutic Application of Genetic Testing}

In the setting of $B R C A$-altered pancreatic cancer, responses to PARP inhibitors can be "profound and durable," she noted.

In the 2019 POLO trial, patients with $B R C A$ mutations who showed a response to platinum-based therapy and then received maintenance olaparib gained a median of 3.5 months (hazard ratio [HR], 0.53) of progression-free survival time. ${ }^{3}$ Overall survival data are not mature, but the tail on the curve suggests durability. The data support using olaparib in this population as an alternative to further intravenous treatment.

Other studies of platinum-based therapy, with and without a PARP inhibitor, further support knowledge germline status at diagnosis. In a recent randomized phase II study of patients with BRCA/PALB2 mutations, the addition of the PARP inhibitor veliparib to cisplatin/gemcitabine did not improve outcomes, but the effect of the platinum was clear in each arm by the very high overall response and disease control rates and robust survival signal. ${ }^{4}$

"The paradigm of sequential platinum followed by a PARP inhibitor is the best way to utilize both approaches [platinum and PARP inhibitor]," Dr. O'Reilly concluded.

A series of trials is building on the use of PARP inhibitors as maintenance. Based on the high rates of chromosomal instability and neoantigen load in BRCA-mutated tumors, an MSK study is adding pembrolizumab to maintenance olaparib, with arms stratified by mutation profile and exceptional response to platinum therapy.

\section{Adjuvant Therapy: Overall Survival Advantage Proven}

Based on the phase III PRODIGE 24/CCTG PA.6 trial, the state-of-the-art adjuvant regimen is mFOLFIRINOX (oxaliplatin $85 \mathrm{mg} / \mathrm{m}^{2}$, leucovorin $400 \mathrm{mg} / \mathrm{m}^{2}$, irinotecan $150 \mathrm{mg} / \mathrm{m}^{2}$, and 5-FU infusion $2.4 \mathrm{~g} / \mathrm{m}^{2}$ over 46 hours, without bolus, given every 2 weeks for 12 cycles). ${ }^{5}$ This regimen was compared with single-agent gemcitabine $1000 \mathrm{mg} / \mathrm{m}^{2}$ for 6 cycles, in a stringently selected patient population.

"What caught our attention was the overall survival data," she said. Modified FOLFIRINOX resulted in longest overall survival reported yet for resected pancreatic cancer: 54.4 months compared with 35.0 months with gemcitabine (HR, 0.64; $P=.003$ ), with median diseasefree survival of 21.6 versus 12.8 months (HR, 0.58; $P<.0001)$, respectively. As expected, toxicity was greater with modified FOLFIRINOX.

"The study was practice-changing, and it led to the routine integration of modified FOLFIRINOX into the adjuvant setting for patients with a good performance status. It clearly matters what's given upfront," Dr. O'Reilly maintained.

For patients who are not candidates for modified FOLFIRINOX, an alternate adjuvant option is gemcitabine plus capecitabine. In ESPAC-4, the doublet yielded a median overall survival of 28 months compared with 25 months with gemcitabine. ${ }^{6}$ An adjuvant regimen that cannot be supported, conversely, is gemcitabine plus albumin-bound (nab)-paclitaxel, based on the disappointing results of the recent APACT trial. ${ }^{7}$ Consensus is lacking for adjuvant chemotherapy plus radiation, which is being studied in the phase III RTOG 0848 trial and for which results are anticipated in 2021.

\section{Surgery First or Neoadjuvant Therapy?}

Another evolving discussion pertains to the use of neoadjuvant therapy for patients with resectable tumors. Upfront surgery, the conventional approach, is associated with a significant risk of relapse, inability to ability 
delivery therapy in a timely way in a significant fraction of patients; the risk is theoretically less with neoadjuvant therapy as it provides early systemic therapy to more patients and selects out the best individuals who will benefit from surgery

The A021806 Alliance Trial will randomize patients with resectable tumors to surgery followed by adjuvant modified FOLFIRINOX (mFOLFIRINOX) or neoadjuvant treatment with mFOLFIRINOX for 8 cycles, followed by surgery and then adjuvant mFOLFIRINOX for 4 cycles.

"By comparing the 2 key frontline approaches in a perioperative and adjuvant, this study will help to optimize our treatment selection for patients with localized disease," Dr. O’Reilly predicted.

\section{Therapy for Advanced Disease}

A standard of care for advanced disease is FOLFIRINOX, based on the survival advantage in the landmark trial. ${ }^{8}$ Gemcitabine/nab-paclitaxel also showed a survival advantage, with acceptable toxicity, in the MPACT trial. ${ }^{9}$

Whether FOLFIRINOX is superior to gemcitabine/ nab-paclitaxel is not clear, as direct comparisons are lacking. A retrospective "real world" study of 654 patients suggests that FOLFIRINOX "has the edge" in terms of outcomes, ${ }^{10}$ "though it's fair to say that gemcitabine/ nab-paclitaxel is suitable for a larger population," she advised. "It is also a platform for the development of experimental agents, though we are starting to see more advanced disease studies building on (modified) FOLFIRINOX."

\section{Maintenance Therapy and \\ Second-Line Regimens}

"Maintenance therapy is a changing space and one where drug development is emerging," she continued. The randomized phase II PRODIGE 35 PANOPTIMOX study provided support for dropping oxaliplatin and reintroducing it on progression, ${ }^{11}$ a finding she called "reassuring," especially for patients developing neuropathy.

Maintenance therapy is also supported by the POLO trial, which evaluated olaparib in patients with germline $B R C A$ mutations, as previously described.

For second-line therapy, NAPOLI-1 found an overall and progression-free survival advantage for nanoliposomal irinotecan + infusional 5-FU and leucovorin, versus 5-FU/leucovorin. ${ }^{12}$ This led to regulatory approval of this triplet several years ago, and it is increasingly used for patients who have received gemcitabine-based therapy frontline. Data for the use of oxaliplatin/fluoropyrimidine combinations in a second-line setting, however, are mixed. ${ }^{13}$
A recommendation for patients starting therapy for advanced disease with mFOLFIRINOX, gemcitabine/nabpaclitaxel would be the second-line option, with possibly nanoliposomal irinotecan/5-FU/leucovorin in the third line. For patients starting with gemcitabine/nab-paclitaxel, a preferred second-line option would be nanoliposomal irinotecan/5-FU/leucorvorin, followed potentially in the third line by FOLFOX (5-FU/leucovorin/oxaliplatin) or capecitabine/oxaliplatin.

\section{Targeting the Stroma and Microenvironment}

Disappointing results were reported recently for attempts to target the stroma and microenvironment. The phase III HALO 301 study of pegylated recombinant human hyaluronidase (PEGPH20), which targets hyaluronan in the stroma, was negative, as overall survival was similar to gemcitabine/nab-paclitaxel alone. ${ }^{14}$ Other large trials reported negative results for the stemness inhibitor napabucasin and for ibrutinib. ${ }^{15}$ "It remains to be seen whether targeting the microenvironment will be productive," she commented.

\section{Promising Drugs in Development}

Hope remains for several agents in development. Devimistat (CPI-613), which targets mitochondrial metabolism, is being evaluated in the frontline setting with mFOLFIRINOX in a phase III trial. Eryaspase is being evaluated in combination with chemotherapy in the second line. Eryaspase consists of L-asparaginase encapsulated inside RBCs, which degrades asparagine, an amino acid that is essential for cancer cell survival and proliferation.

Immunotherapy may also prove beneficial in the subset of patients with mismatch repair deficiency, although there are challenges. Strategies for overcoming the pancreas tumor's immunosuppressive microenvironment are being evaluated, such as combining nivolumab with chemotherapy and an anti-CD40 antibody. "Meanwhile, it is still an open question as to where immunotherapy fits in this disease," Dr. O'Reilly commented.

Disclosures: Dr. O'Reilly has disclosed that she has received consulting fees from Celgene Corporation, CytomX Therapeutics, Loxo Oncology, Merck \& Co., Inc., Silenseed, Polaris, Ipsen, Rafael Therapeutics, Sobi, and Pharmaceuticals, and her spouse has received consulting fees from Agios, Inc., AstraZeneca Pharmaceuticals LP, Bayer HealthCare, BeiGene, Berry Genomics, Bristol-Myers Squibb Company, Celgene Corporation, Debio, Eisai Inc., Eli Lilly and Company, Exelixis Inc., Flatiron Health, Inc. Genoscience Pharma, Incyte Corporation, Ipsen, LAM Research, Merck \& Co. Inc., MINA, QED, Redhill Biopharma Ltd., SillaJen, twoXAR Pharmaceuticals, and Yiviva.

Correspondence: Eileen M. O'Reilly, MD, Memorial Sloan Kettering Cancer Center, 300 East 66th Street, New York NY 10065.

Email: oreillye@mskcc.org 


\section{References}

1. Lowery MA, Wong W, Jordan EJ, et al. Prospective evaluation of germline alterations in patients with exocrine pancreatic neoplasms. J Natl Cancer Inst 2018;110:1067-1074.

2. Hu ZI, Shia J, Stadler ZK, et al. Evaluating mismatch repair deficiency in pancreatic adenocarcinoma: challenges and recommendations. Clin Cancer Res 2018;24:1326-1336.

3. Golan T, Hammel P, Reni M, et al. Maintenance olaparib for germline BRCA-mutated metastatic pancreatic cancer. N Engl J Med 2019;381: 317-327.

4. O'Reilly EM, Lee JW, Zalupski M, et al. Randomized, multicenter, phase II trial of gemcitabine, cisplatin +/- veliparib in patients with pancreas adenocarcinoma and a known germline BRCA/PALB2 mutation [abstract]. Presented at the 2020 Gastrointestinal Cancers Symposium; January 23-25, 2020; San Francisco, California. Abstract 639

5. Conroy T, Hammet P, Hebbar M, et al. Unicancer GI PRODIGE 24/CCTG PA.6 trial: a multicenter international randomized phase III trial of adjuvant mFOLFIRINOX versus gemcitabine in patients with resected pancreatic ductal adenocarcinomas [abstract]. J Clin Oncol 2018;36(Suppl):Abstract LBA4001.

6. Neoptolemoa JP, Palmer DH, Ghaneh P, et al. Comparison of adjuvant gemcitabine and capecitabine with gemcitabine monotherapy in patients with resected pancreatic cancer (ESPAC-4): a multicentre, open-label, randomised, phase 3 trial. Lancet 2017;389:1011-1024.

7. Tempero MA, Reni M, Riess H, et al. APACT: phase III, multicenter, international, open-label, randomized trial of adjuvant nab-paclitaxel plus gemcitabine vs gemcitabine for surgically resected pancreatic adenocarcinoma [abstract]. Presented at the 2019 ASCO Annual Meeting; May 31-June 4, 2019; Chicago; Illinois. Abstract 4000.

8. Conroy $T$, Desseigne $F$, Ychou M, et al. FOLFIRINOX versus gemcitabine for metastatic pancreatic cancer. N Engl J Med 2011; 364:1817-1825.
9. Von Hoff DD, Envin T, Arena FP, et al. Increased survival in pancreatic cancer with nab-paclitaxel plus gemcitabine. N Engl J Med 2013;369:1691-1703.

10. Kim S, Signorovitch JE, Yang $\mathrm{H}$, et al. Comparative effectiveness of nabpaclitaxel plus gemcitabine vs FOLFIRINOX in metastatic pancreatic cancer: a retrospective nationwide chart review in the United States. Adv Ther 2018;35:1564-1577.

11. Dahan L, Phelip JM, Le Malicot K, et al. FOLFIRINOX until progression, FOLFIRINOX with maintenance treatment, or sequential treatment with gemcitabine and FOLFIRI.3 for first-line treatment of metastatic pancreatic cancer: a randomized phase II trial (PRODIGE 35-PANOPTIMOX) [published online June 1, 2018]. J Clin Oncol, doi: 10.1200/JCO.2018.36

12. Wang-Gillam A, Li CP, Bodoky G, et al. Nanoliposomal irinotecan with fluorouracil and folinic acid in metastatic pancreatic cancer after previous gemcitabine-based therapy (NAPOLI-1): a global, randomized, openlabel, phase 3 trial. Lancet. 2016;387:545-557.

13. Gill S, Ko YJ, Cripps C, et al. PANCREOX: a randomized phase III study of fluorouracil/leucovorin with or without oxaliplatin for second-line advanced pancreatic cancer in patients who have received gemcitabinebased chemotherapy. J Clin Oncol 2016;34:3914-3920.

14. Tempero MA, Van Cutsem E, Sigal D, et al. HALO 109-301: a randomized double-blind, placebo-controlled, phase III study of pegvorhyaluronidase alfa (PEGPH2O) + nab-paclitaxel/gemcitabine in patients with previously untreated hyaluronan-high metastatic pancreatic ductal adenocarcinoma [abstract]. Presented at the 2020 Gastrointestinal Cancers Symposium: January 23-25, 2020; San Francisco, California. Abstract 638.

15. Boston Biomedical. Boston Biomedical, Inc. announces update on phase 3 CANSTE111P study of napabucasin in patients with metastatic pancreatic cancer following interim analysis. Accessed March 20, 2020. Available at: https://www.bostonbiomedical.com/news-and-media/ 20190701_boston-biomedical-inc-announces-update-canstem111pstudy-following-interim-analysis/ 